\title{
Registro del Grisón (Galictis vittata Schreber, 1776) en un humedal de Alvarado, Veracruz, México
}

\author{
Julio C. Hernández-Hernández*i(i), Elio Lagunes-Díaz1(i) \\ 1 Pronatura Veracruz A.C. Ignacio Zaragoza 153, Centro, C.P. 91500, Veracruz, México. \\ * Correspondencia:jhernandez@pronaturaveracruz.org
}

\section{Resumen}

Presentamos un nuevo registro de la presencia del Grisón (Galictis vittata) en el sureste de México. Este registro se obtuvo a partir de cámaras-trampa en un Área Privada de Conservación. Adicionalmente, se analizó la literatura científica y bases de datos especializadas con la finalidad de corroborar los registros previos de esta especie para México. Este registro contribuye al conocimiento de la distribución geográfica del Grisón para la región sureste de México, por lo que se refuerza la idea de la importancia de implementar programas de monitoreo y conservación.

Palabras clave: Cámaras-trampa, Distribución, Mustelidae, Sureste de México

\begin{abstract}
We present a new record of the presence of the grison (Galictis vittata) in the southeast of Mexico. This record was obtained from camera traps in a Private Conservation Area. Additionally, the scientific literature and specialized databases were analyzed in order to corroborate the previous records of this species for Mexico. This record also constitutes a contribution to the knowledge of the geographical distribution of grison for the southeast region of Mexico, thus reinforcing the idea of the importance of implementing monitoring and conservation programs.
\end{abstract}

Key words: Camera-traps, Distribution, Mustelidae, Southeast Mexico.

El Grisón, Galictis vittata (Shreber 1776), es una especie de la familia Mustelidae que se distribuye a lo largo de las áreas tropicales y subtropicales de América. El límite norte de su distribución es el sur del estado de Tamaulipas, México, extendiéndose a través de Centroamérica hasta el norte de Argentina, el sur de Bolivia y Brasil (Yensen \& Tarifa 2003; Contreras-Díaz et al. 2020). Esta especie se distribuye desde el nivel del mar hasta los 1.500 msnm, aunque la mayoría de los registros se encuentran por debajo de los $500 \mathrm{msnm}$ (Yensen \& Tarifa 2003). En Colombia existe un registro de Grisón a los 2.200 msnm, lo que establece el sitio con mayor altura reportado para esta especie (Escobar-Lasso \& GuzmánHernández 2014).

Su dieta está compuesta principalmente de mamíferos, sin embargo, reptiles y anfibios como lagartijas, serpientes, ranas y sapos también forman parte de su dieta (Bisbal 1986; 
Cintra 1988; Sunquist et al. 1989; Yensen \& Tarifa 2003; Hidalgo-Mihart et al. 2018). Los principales tipos de vegetación en los que se distribuye son bosques tropicales perennifolios, subperennifolios y semideciduos (Chávez 2005), frecuentemente son encontrados cerca de los ríos, arroyos y pantanos (Emmons \& Feer 1999; Yensen \& Tarifa 2003).

A pesar de que existen registros del Grisón para el estado de Veracruz, México (Gallina et al. 1996; González-Christen 2008; González-Christen \& Coates 2019), no existía evidencia sobre su presencia dentro del Sistema Lagunar de Alvarado (SLA), el cual se caracteriza por ser uno de los ecosistemas con los bosques de manglar más extensos del estado de Veracruz, con más de 19.000 ha (Franco-López 2017). Así también, el SLA es uno de los humedales más representativos del estado por su riqueza biológica, su gran potencial productivo, y por su arraigada tradición cultural, cuyo eje central es la actividad pesquera (Portilla-Ochoa et al. 2007). Además, fue declarado sitio Ramsar en el 2004, por su importancia y la biodiversidad de sus humedales (Portilla-Ochoa 2003).

Como parte del monitoreo de la biodiversidad del Área Privada de Conservación (APC) "Cala Larga", ubicado en la zona centro del Sistema Lagunar de Alvarado, Veracruz, México, se realizó un muestreo sistemático con fototrampeo durante enero a diciembre del año 2016. Se instalaron 44 estaciones con una trampa cámara (Moultrie A-35) de forma aleatoria a una altura de $30 \mathrm{~cm}$ a $40 \mathrm{~cm}$ del suelo, en sitios donde se detectaron rastros de mamíferos silvestres (e. g. huellas, excretas, senderos), con una separación entre cada estación de 1 a $3 \mathrm{~km}$ para no dejar grandes vacíos sin muestrear. Las cámaras fueron instaladas en sitios donde predominan tres especies de mangle: mangle blanco (Laguncularia racemosa), mangle rojo (Rhizophora mangle) y mangle negro (Avicennia germinans). Las cámaras permanecieron activas las 24 horas del día y estuvieron programadas para tomar una fotografía y un video de 30 segundos, con la fecha y hora de cada registro.

Mediante este esquema, se obtuvo el registro de un individuo de Grisón el día 18 de diciembre de 2016 a las 15h42 (18,707150, -95,766308; 6 msnm; Figura 1), con un esfuerzo de muestreo de 337 días efectivos, dentro de vegetación asociada al manglar (Randia aculeata, Lonchocarpus luteomaculatus, Zygia latifolia), lo que coincide con observaciones previas en los humedales Laguna de Términos y Pantanos de Centla en Tabasco, México (Hidalgo-Mihart et al. 2017) y la Reserva de la Biosfera la Encrucijada, Chiapas, México (Hernández-Hernández et al. 2018). Además, con la finalidad de corroborar los registros previos de esta especie para México, se consultó la información disponible en Global Biodiversity Information Facility (GBIF 2019) y en el Sistema Nacional de Información sobre Biodiversidad (SNIB 2020), correspondientes al periodo comprendido entre 1879 y 2020, siendo el más cercano el de la localidad de Potrero Viejo, en el Municipio de Amatlán de los Reyes, Veracruz, a 100 km del presente registro (Figura 2) (Slade 2020).

El video muestra las características morfológicas comunes de la especie: coloración dorsal jaspeada, con una franja blanca desde la frente a los hombros, que pasa por debajo de las orejas y arriba de los ojos, región ventral y patas negras, extremidades y cola cortas (Álvarez-Castañeda et al. 2015).

En México, el Grisón es considerado como una especie amenazada en la ley de protección de especies de flora y fauna (SEMARNAT 2010). Igualmente, se encuentra incluido entre las especies de mayor prioridad nacional para la conservación de carnivoros terrestres mexicanos (Valenzuela \& Vásquez 2007). Además es considerada una rareza demográfica, 
ya que tiene una densidad baja a lo largo de su intervalo de distribución (Yensen \& Tarifa 2003; Cuarón et al. 2016).

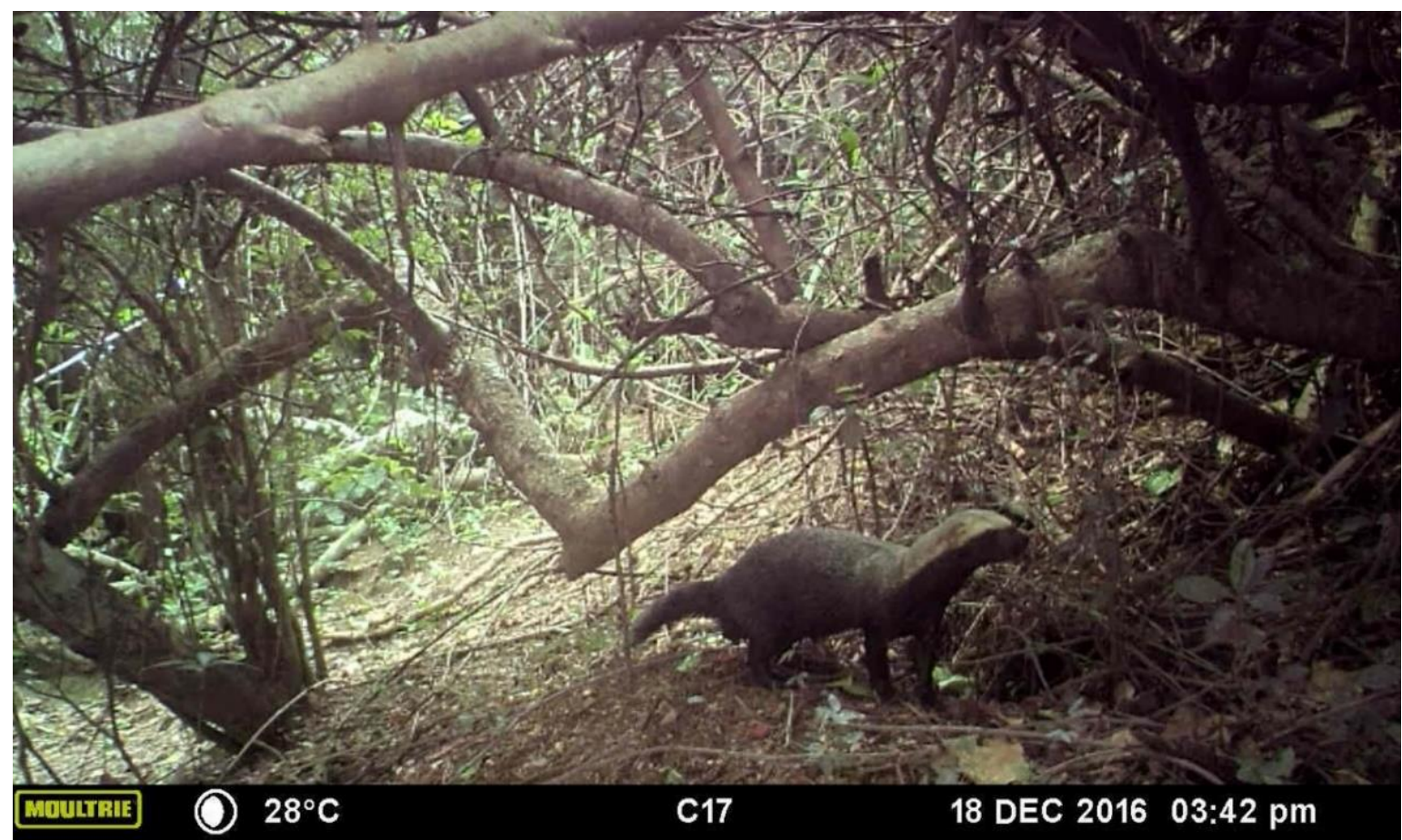

FIGURA 1. Registro del Grison (Galictis vittata) en el Área Privada de Conservación "Cala Larga", Alvarado, Veracruz, México.

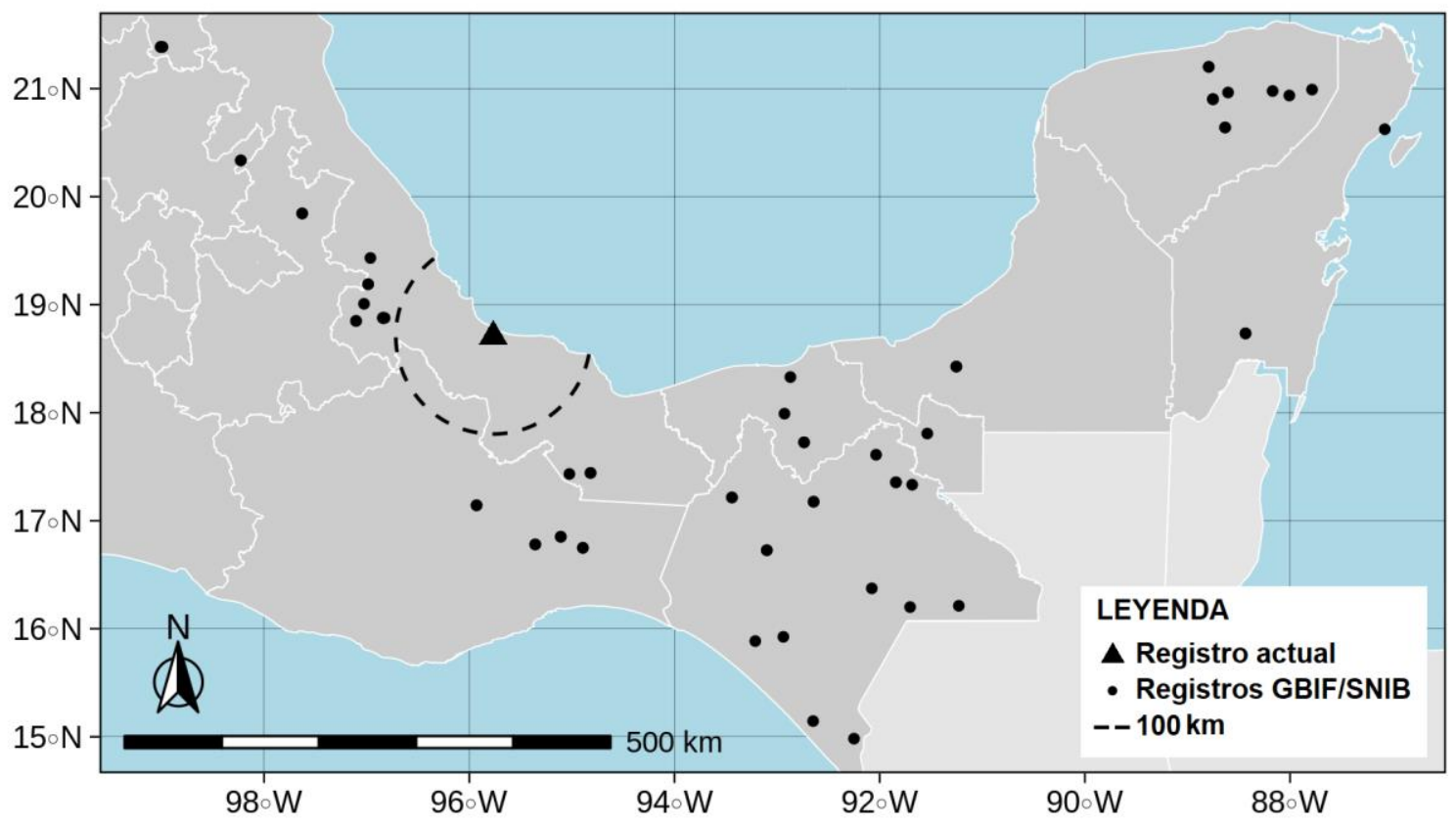

FIGURA 2. Localización geográfica de los registros del Grison (Galictis vittata) en México (GBIF 2019; SNIB 2020). 
Este registro es evidencia de la presencia del Grisón en el litoral Veracruzano, y contribuye al conocimiento de su distribución en México. Contar con información actualizada sobre la distribución de esta especie es fundamental para planificar su conservación, teniendo en cuenta la fuerte transformación de los humedales en el país y en el estado de Veracruz (Moreno-Casasola et al. 2010). Adicionalmente en la zona de estudio predominan tres especies de mangle consideradas como Amenazadas en la legislación ambiental vigente en México (SEMARNAT 2010). Finalmente, es indispensable realizar más investigaciones en la región con el fin de conocer las amenazas y su densidad poblacional, de modo que constituyan la base para implementar estrategias de monitoreo y conservación.

\section{AGRADECIMIENTOS}

Agradecemos el financiamiento de la Germany's International Climate Initiative (IKI) y la North American Wetlands Conservation Act (NAWCA) por medio del U.S. Fish and Wildlife Service.

\section{REFERENCIAS}

Álvarez-Castañeda ST, Álvarez S, González-Ruiz N. 2015. Guía para la identificación de los mamíferos de México en campo y laboratorio. Centro de Investigaciones Biológicas del Noroeste, S. C. y Asociación Mexicana de Mastozoología, A. C.

Bisbal FJ. 1986. Food habits of some Neotropical carnivores in Venezuela (Mammalia, Carnivora). Mammalia 50:329-339. https:// doi.org/10.1515/mamm.1986.50.3.329

Chávez, C. 2005. Galictis vittata. In: Ceballos G, Oliva G, editors. Los mamíferos de México. Comisión Nacional para el Conocimiento y Uso de la Biodiversidad, Fondo de Cultura Económica. México. p. 378-380

Cintra R. 1988. Bufo marinus (marine toad) predation. Herpetology Review 19:82.

Contreras-Díaz C, Soria-Díaz L, Astudillo-Sánchez CC, Domínguez-Vega H, Gómez-Ortiz Y, MartínezGarcía I. 2020. Expansion of distribution range of the greater grison (Galictis vittata) in Mexico. Therya notes 1:1-4. https:// doi.org/10.12933/therya_notes-20-1

Cuarón AD, Reid F, González-Maya, JF, Helgen K. 2016. Galictis vittata. The IUCN Red List of Threatened Species 2016. https:// dx.doi.org/10.2305/IUCN.UK.2016-1.RLTS.T41640A45211961.en

De la Torre J, Muech C, Arteaga M. 2009. Nuevos registros de grisón (Galictis vittata) para la selva Lacan-dona, Chiapas, México. Revista Mexicana de Mastozoología 13:109-114. http:// dx.doi.org/10.22201/ie.20074484e.2009.13.1.40

Escobar-Lasso S, Guzmán-Hernández C. 2014. El registro de mayor altitud del Hurón Mayor Galictis vittata, con notas sobre su presencia y conservación dentro del departamento de Caldas, en la región andina de Colombia. Therya 5:567-573. https:// doi.org/10.12933/therya-14-166

Franco-López J. 2017. Análisis del comportamiento estacional e interanual de la comunidad ictiofaunística asociada a zonas de manglar en la laguna de Alvarado, Veracruz. BIOCYT Biología, Ciencia y $\quad$ 10:722-735. http:/ / dx.doi.org/10.22201/fesi.20072082.2017.10.61475

Gallina S, Mandujano S, González-Romero A. 1996. Conservation of mammalian biodiversity in coffee plantations of Central Veracruz, Mexico. Agroforestry Systems 33:13-27. https://doi.org/10.1007/BF00122886 
GBIF. 2019. Global Biodiversity Information Facility. Occurrence download. https://www.gbif.org/species/5218906. Accessed on 28 July 2020.

González-Christen A. 2008. La diversidad, alfa, beta y gama de la mastofauna en la Sierra de Santa Marta, Veracruz, México. In: Lorenzo C, Espinoza E, Ortega J, editores. Avances en el estudio de los mamíferos de México, Volumen II. Asociación Mexicana de Mastozoología A. C. Ciudad de México, México. p. 103-123.

González-Christen A, Coates R. 2019. Los mamíferos no voladores de la región de Los Tuxtlas, Veracruz, México. Revista Mexicana de Biodiversidad 90: e902580. http://dx.doi.org/10.22201/ib.20078706e.2019.90.2580

Hernández-Hernández JC, Ruiz-Gutiérrez F, Vázquez-Arroyo E, Chávez C. 2018. Registros del grisón (Galictis vittata), nutria de río neotropical (Lontra longicaudis) y conejo (Sylvilagus sp) en la Reserva de la Biosfera La Encrucijada, Chiapas, México. Revista Mexicana de Mastozoología nueva época 8:8-12. http:/ / dx.doi.org/10.22201/ie.20074484e.2018.1.1.253

Hidalgo-Mihart MG, Contreras-Moreno FM, Jesús-de la Cruz A, Juárez-López R, Bravata de la Cruz Y, Pérez-Solano LA, Hernández-Lara C, Friedeberg D, Thornton D, Koller-González JM. 2017. Inventory of medium-sized and large mammals in the wetlands of Laguna de Terminos and Pantanos de Central, Mexico. Check List 13:711-726. https:// doi.org/10.15560/13.6.711

Hidalgo-Mihart MG, Juárez-López R, Contreras-Moreno F, De la Cruz AJ, López-Luna MA, Bravata de la Cruz Y. 2018. Greater grison (Galictis vittata) hunts a central american indigo snake (Drymarchon melanurus) in Southeastern Mexico. The Southwestern Naturalist 63:197-199. https:// doi.org/10.1894/0038-4909-63-3-197

Moreno-Casasola P, Infante-Mata D, Sánchez-Vigil G. 2010. Veracruz. Tierra de ciénagas y pantanos. Gobierno del Estado de Veracruz, Secretaría de Educación del Estado de Veracruz.

Portilla-Ochoa E (comp.). 2003. Ficha informativa de los humedales de Ramsar "Sistema Lagunar Alvarado" . The Ramsar Convention on Wetlands y Comisión Nacional de Áreas Naturales Protegidas. Xalapa, Veracruz, México.

Sánchez EA. 2015. Caracterización de tres propiedades del suelo en humedales transformados a potreros, en el municipio de Jamapa, Veracruz y su entorno. Tesis de Especialidad. Facultad de Ciencias Químicas, Universidad Veracruzana, Xalapa. México.

SEMARNAT. Secretaría de Medio Ambiente y Recursos Naturales. 2010. Norma Oficial Mexicana NOM059-SEMARNAT-2010. MODIFICACIÓN del Anexo Normativo III, Lista de especies en riesgo de la Norma Oficial Mexicana NOM-059-SEMARNAT-2010, Protección ambiental-Especies nativas de México de flora y fauna silvestres-Categorías de riesgo y especificaciones para su inclusión, exclusión o cambio-Lista de especies en riesgo, publicada el 30 de diciembre de 2010. Accessed on 27 july 2020.

SNIB. Sistema Nacional de Información Sobre Biodiversidad. 2020. Registros de ejemplares. Comisión Nacional para el Conocimiento y Uso de la Biodiversidad. Ciudad de México, México. http:/ / www.snib.mx. Accessed on 28 july 2020.

Slade N. 2020. KUBI Mammalogy Collection. Version 26.40. University of Kansas Biodiversity Institute. https:// doi.org/10.15468/a3woj7 Accessed on 22 December 2020.

Sunquist ME, Sunquist F, Daneke DE. 1989. Ecological separation in a Venezuelan llanos carnivore com-munity. In: Redford KH, Eisenberg JF, editores. Advances in Neotropical Mammalogy. Sandhill Crane Press, Gainesville, Florida. p. 197-232.

Valenzuela D, Vásquez LB. 2007. Consideraciones para priorizar la conservación de carnívoros mexicanos. In: Sánchez-Rojas G, Rojas-Martínez A, editores. Tópicos en sistemática, 
biogeografía, ecología y conservación de mamíferos. Universidad Autónoma del estado de Hidalgo. p. 197-214

Yensen E, Tarifa T. 2003. Galictis vitatta. Mammalian Species 727:1-8. https:// doi.org/10.1644/0.727.1

Editor: Diego J. Lizcano

Recibido 2020-12-01

Revisado 2021-01-10

Aceptado 2021-04-01

Publicado 2021-04-05 